\title{
Regular Solutions in Vacuum Brans-Dicke Theory Compared to Vacuum Einstein Theory
}

\author{
Alina Khaybullina, ${ }^{1}$ Ramil Izmailov, ${ }^{1}$ Kamal K. Nandi, ${ }^{1,2}$ and Carlo Cattani ${ }^{1,3}$ \\ ${ }^{1}$ Ya.B. Zel'dovich International Center for Astrophysics, BSPU, Ufa 450000, Russia \\ ${ }^{2}$ Department of Mathematics, University of North Bengal, Siliguri 734013, India \\ ${ }^{3}$ Department of Mathematics, University of Salerno, Via Ponte Don Melillo, 84084 Fisciano, Italy
}

Correspondence should be addressed to Carlo Cattani; ccattani@unisa.it

Received 28 June 2013; Accepted 24 August 2013

Academic Editor: Gongnan Xie

Copyright (C) 2013 Alina Khaybullina et al. This is an open access article distributed under the Creative Commons Attribution License, which permits unrestricted use, distribution, and reproduction in any medium, provided the original work is properly cited.

\begin{abstract}
We will confront some static spherically symmetric vacuum Brans-Dicke solutions in the Jordan and Einstein Frames with the Robertson parameters. While the regular solution in the vacuum Einstein theory is just the Schwarzschild black hole, the same in the Jordan frame Brans-Dicke theory is shown to represent not a black hole but a traversable wormhole. But, in this case, the valid range of $\omega$ becomes too narrow to yield the observed weak field Robertson parameters at the positive mass mouth. The corresponding solution in the Einstein frame also provides a regular wormhole, and it yields the correct parametric values but only up to "one and half order." We argue that a second-order contribution can in principle distinguish between the signatures of the regular wormhole and the singular Buchdahl solution in the Einstein frame. Thus, at the level of regular solutions, Brans-Dicke theory in each frame predicts effects very different from those of Einstein's theory. To our knowledge, these theoretical distinctions seem not to have received adequate attention so far.
\end{abstract}

\section{Introduction}

It is known from history that Einstein was much influenced by Mach's principle ${ }^{1}$ and that he tried to incorporate the principle in his theory of general relativity. The principle has many variants, but within the field theory framework, the simplest one is that a static distribution of matter should cause only a static geometry. However, Gödel [1] in 1949 showed that this is not the case: Einstein's general relativity equations produce a rotating universe even though the matter distribution is static! The incorporation of Mach's principle in a field theory framework was accomplished by Brans and Dicke in 1961 [2]. There has been a lot of important works in wormholes and cold black hole spacetimes initiated by Bronnikov et al. [3-5] earlier, Brans et al. listed four classes of static spherically symmetric solutions in their theory [6]. On the other hand, enormous work in the literature exists exploring various features of the Brans-Dicke (BD) theory. However, most of the works dealing with spherically symmetric solutions in the BD theory concentrate only on the singular Brans Class I solution, and nothing is asked if regular solutions exist at all in the theory. However, the singularity in Brans Class I solution for unrestricted $\omega$ should be mandatorily admitted if one wants the solution to test against the weak field solar system tests.

The BD field equations as well as the Class I solution yield post-Newtonian $(\mathrm{pN})$ expansion that coincides at $|\omega| \rightarrow \infty$ with the same expansion of Einstein's field equations and so the latter has gained popularity as the local solution. The fact that it has a naked singularity is noted, but the solution is not abandoned as unphysical. Moreover, the current limit on the coupling constant $|\omega| \geq 50000$ creates the impression that BD theory no longer stands as a distinct alternative to Einstein's theory. However, in this context, we mention that the belief that Einstein's theory is recovered from BD theory at $|\omega| \rightarrow$ $\infty$ is not always true, but we will not discuss it here (see, for details, [7]).

If we want the naked singularity in Class I solution to disappear, additional inequalities connecting the integration constants $C$ and $\lambda$ must be introduced reducing the solution 
to what were termed as cold black hole spacetimes. Cold because the Hawking temperature is zero. This leads to several difficulties on physical grounds. First, the inequality severely restricts the range of $\omega$ to an absurdly limited range so that the weak field tests are out of question. Second, although coordinate patch can be extended beyond the $c b h$ horizon, it leads to disruption of freely falling particles caused by large tidal forces just above the horizon. The $c b h$ then becomes naked black hole a la Horowitz and Ross. For Campanellu Lousto $\mathrm{BH}$ condition, namely, $C+1 \geq 2 \lambda$, all curvature invariants vanish at $r=B$. With $C=-1 /(\omega+2)$, this implies a very restrictive range $-(2+1 / \sqrt{3})<\omega<-2$, while $r=B$ being an outgoing null surface requires $C+2>\lambda \Rightarrow \omega<-2$. Excess tidal forces become finite if $\xi=C / 2 \lambda>1$, but Hawking temperature finite at $r=B$ requires $C / 2 \lambda<1$. $a=(1 / \lambda)((C+2) / 2)$. For the range $-(2+1 / \sqrt{3})<\omega<-2$, we see that $\xi=C / 2 \lambda<1$; hence, tidal forces tear apart a test body. But solar tests are out of question.

For regular Class II solution, which is a wormhole, weak field tests are not possible because $-2<\omega<-3 / 2$, but tidal forces are finite at the throat.

The question is if all observed tests boil down to only tallying with the $\mathrm{pN}$ or for that matter model independent Robertson parameters, what is the need for dealing with exact solutions at all in both the theories? The answer is that the exact solutions offer richer, and testable, geometric structures of black holes, naked singularities, wormholes, and so forth. It is along this thought that we want to investigate one $\mathrm{BD}$ regular solution and its conformal version and ask what kind of geometry it represents, and where exactly it differs from naked singularities or black holes. Previously, it was reported in the literature (see, for details, $[8,9]$ ) that the desired regular solution of the vacuum BD theory in the Jordan Frame (JF) as well as in the conformally rescaled Einstein Frame (EF) represents traversable wormhole geometries. ${ }^{2}$

Our purpose here is to confront the singular and regular solutions with the generic Robertson parameters. We argue how a second-order contribution to deflection can in principle differentiate between a wormhole and a singular solution in the EF. The practical problems about measuring the second-order contribution in model independent situation are also addressed briefly. The general conclusion is that those regular natural wormhole spacetimes would never reduce at any limit to Schwarzschild black hole of the vacuum Einstein theory. (We take $G=1, c=1$.)

\section{Brans Class I Singular Solution in JF}

We begin by starting with the Robertson expansion for a centrally symmetric configuration in isotropic coordinates given by $[10,11]$

$$
\begin{aligned}
d \tau^{2}= & -B(r) d t^{2}+A(r) \\
& \times\left[d r^{\prime 2}+r^{2}\left(d \theta^{2}+\sin ^{2} \theta d \psi^{2}\right)\right]
\end{aligned}
$$

$$
\begin{aligned}
= & -\left(1-2 \alpha_{1} \frac{M}{r}+2 \beta_{1} \frac{M^{2}}{r^{2}}-\frac{3}{2} \epsilon_{1} \frac{M^{3}}{r^{3}}+\cdots\right) d t^{2} \\
& +\left(1+2 \gamma_{1} \frac{M}{r}+\frac{3 \delta_{1} M^{2}}{2 r^{2}}+\cdots\right) \\
& \times\left[d r^{2}+r^{2}\left(d \theta^{2}+\sin ^{2} \theta d \psi^{2}\right)\right],
\end{aligned}
$$

where $\alpha_{1}, \beta_{1}, \epsilon_{1}, \gamma_{1}$, and $\delta_{1}$ are constant parameters to be found by fitting with observations. As well known, Brans Class I solution [6] in the JF is given in isotropic coordinates $(t, r, \theta, \psi)$ by

$$
\begin{aligned}
d \tau^{2}= & -e^{2 \alpha(r)} d t^{2}+e^{2 \beta(r)} \\
& \times\left[d r^{2}+r^{2}\left(d \theta^{2}+\sin ^{2} \theta d \psi^{2}\right)\right], \\
& e^{\alpha(r)}=e^{\alpha_{0}}\left[\frac{1-B / r}{1+B / r}\right]^{1 / \lambda}, \\
e^{\beta(r)}= & e^{\beta_{0}}\left[1+\frac{B}{r}\right]^{2}\left[\frac{1-B / r}{1+B / r}\right]^{(\lambda-C-1) / \lambda}, \\
& \varphi(r)=\varphi_{0}\left[\frac{1-B / r}{1+B / r}\right]^{C / \lambda} .
\end{aligned}
$$

When this solution is put in the BD field equations, it yields the constraint equation as

$$
\lambda^{2} \equiv(C+1)^{2}-C\left(1-\frac{\omega C}{2}\right)>0,
$$

where $\lambda, \alpha_{0}, \beta_{0}, B, C$, and $\varphi_{0}$ are real constants. The constants $\alpha_{0}$ and $\beta_{0}$ are determined by asymptotic flatness at $r=+\infty$ as $\alpha_{0}=\beta_{0}=0$.

Under the usual Newtonian limit giving $M=2 B / \lambda$ and $C(\omega)=-(1 /(\omega+2))$, the above solution expands such that $\alpha_{1}=1, \beta_{1}=1$, and $\gamma_{1}=(\omega+1) /(\omega+2)$. For finite $\omega$, the solution has a naked singularity at $r=B$. Further, the constraint (3) yields

$$
\lambda= \pm \sqrt{\frac{2 \omega+3}{2 \omega+4}} .
$$

The negative $\lambda$ is ruled out as it makes $e^{\alpha(r)}$ singular at $r=B$ and the mass $M$ negative. The solutions (2) and (3) with the positive value of $\lambda$, even though singular, immediately yield the above weak field Robertson expansion (1) that depends on arbitrary values of $\omega$. Indeed even the limit $\omega \rightarrow \pm \infty$ is possible, making the solution eventually indistinguishable from the Einstein values $\alpha_{1}=1, \beta_{1}=1$, and $\gamma_{1}=1$, and of course from (4) the ultimate Schwarzschild limit $\lambda=1$, $C=0$ is achieved. We know that the Schwarzschild solution is a regular black hole solution in Einstein's theory. A natural question is to ask whether or not we can find a regular solution in the JF Brans-Dicke theory. The answer is we can, and it is exactly this regular solution that makes the passage to Schwarzschild limit impossible. 


\section{Regular Wormhole in JF}

The regular solution can be obtained from the Class I solution by the following Wick-like rotations on it:

$$
\begin{gathered}
r \longrightarrow \frac{1}{r^{\prime}}, \quad B \longrightarrow \frac{i}{B^{\prime}}, \quad \lambda \longrightarrow-i \Lambda, \\
\alpha_{0} \longrightarrow \epsilon_{0}, \quad \beta_{0} \longrightarrow \delta_{0}+2 \ln B^{\prime},
\end{gathered}
$$

where $B^{\prime}$ and $\Lambda$ are real. Using the identity

$$
\tan ^{-1}(x)=\frac{i}{2} \ln \left(\frac{1-i x}{1+i x}\right),
$$

we arrive at the metric functions and the scalar field as follows:

$$
\begin{aligned}
d \tau^{2}= & -e^{2 \alpha\left(r^{\prime}\right)} d t^{2}+e^{2 \beta\left(r^{\prime}\right)} \\
& \times\left[d r^{\prime 2}+r^{\prime 2}\left(d \theta^{2}+\sin ^{2} \theta d \psi^{2}\right)\right],
\end{aligned}
$$

where

$$
\begin{gathered}
\alpha\left(r^{\prime}\right)=\epsilon_{0}+\frac{2}{\Lambda} \tan ^{-1}\left(\frac{r^{\prime}}{B^{\prime}}\right) \\
\beta\left(r^{\prime}\right)=\delta_{0}-\frac{2(C+1)}{\Lambda} \tan ^{-1}\left(\frac{r^{\prime}}{B^{\prime}}\right)-\ln \left(\frac{r^{\prime 2}}{r^{\prime 2}+B^{\prime 2}}\right), \\
\varphi\left(r^{\prime}\right)=\varphi_{0} \exp \left[\frac{2 C}{\Lambda} \tan ^{-1}\left(\frac{r^{\prime}}{B^{\prime}}\right)\right] \\
\Lambda^{2} \equiv C\left(1-\frac{\omega C}{2}\right)-(C+1)^{2}>0
\end{gathered}
$$

Asymptotic flatness at $r^{\prime}=\infty$ requires that

$$
\epsilon_{0}=-\frac{\pi}{\Lambda}, \quad \delta_{0}=\frac{\pi(C+1)}{\Lambda} .
$$

The above solution set is regular everywhere including at $r^{\prime}=$ $B^{\prime}$ and is an exact nonsingular traversable wormhole solution (see, for details, [9]). Incidentally, Brans [6] mentioned the above solution as Class II among his list of four classes but is not recognized as such. ${ }^{3}$ However, though not independent, we see that Class I and II solutions are fundamentally different as the former is singular, while the latter is a regular wormhole. Does there anyway exist a transition from this regular Brans-Dicke to Schwarzschild solution under $\omega \rightarrow$ $\pm \infty$ ? No, as we show below.

It can be verified that the weak field expansions of $e^{2 \alpha\left(r^{\prime}\right)}$, using relevant identities, yield, respectively, the Schwarzschild masses $M^{ \pm}$

$$
\begin{gathered}
M^{+}=\frac{2 B^{\prime}}{\Lambda} \quad \text { as } r^{\prime} \longrightarrow+\infty, \\
M^{-}=-\frac{2 B^{\prime}}{\Lambda} \exp \left[\frac{2 \pi}{\Lambda}\right] \quad \text { as } \widehat{r} \longrightarrow-\infty,
\end{gathered}
$$

of two asymptotically flat spacetimes on two different coordinate charts lying on either side of the minimal circumference (wormhole throat). The solution in the positive mass $\left(2 B^{\prime} / \Lambda\right)$ side formally expands exactly as desired in the Robertson expansion (1) leading to parameter values $\alpha_{1}=\beta_{1}=1$, $\gamma_{1}=(\omega+1) /(\omega+2)$, and $\delta_{1}=\left(\omega^{4}+23 \omega+14\right) / 6(\omega+2)^{2}$, similar to those of singular solution of Section 2. But the story is not complete yet. Note that we have not yet specified the range of values of $\omega$. With $C=-(1 /(\omega+2))$, this range will come from the constraint (3), namely,

$$
\Lambda=\sqrt{-\left(\frac{2 \omega+3}{2 \omega+4}\right)}
$$

so that

$$
M^{+}=\frac{2 B^{\prime}}{\sqrt{-((2 \omega+3) /(2 \omega+4))}} .
$$

Now $\Lambda$ has to be real in order for the mass $M^{+}$to be real, and it is exactly from here that a narrow range for $\omega$ appears, namely, $-2<\omega<-3 / 2$. Consequently, it does not yield the desired value of positive $\gamma_{1} \sim 1$, hence inconsistent with solar system tests. Even the possibility $\omega \rightarrow \pm \infty$ is ruled out in this case since $\Lambda$ and $M^{+}$become imaginary. Alternatively, we may allow $B^{\prime}$ and $\Lambda$ to be imaginary to keep $M^{+}$real, but then we have to sacrifice regularity because we would get back the same singular Class I solution as soon as these imaginary quantities are put into (5) and (11). Hence, in the JF, the regular Brans-Dicke solution does not explain solar system tests nor does it go over to Schwarzschild black hole (no horizon). It is just a regular wormhole born as such and existing ever since. This is the price of having a regular BD solution! We now concentrate on the picture in the EF.

\section{Buchdahl Singular Solution in EF}

The picture in the EF is completely different due to the fact that $\omega$ does not appear in this frame. Under the substitution

$$
\begin{gathered}
\tilde{g}_{\mu \nu}=\varphi g_{\mu \nu}, \\
d \phi=\sqrt{\frac{2 \omega+3}{2 \mu}} \frac{d \varphi}{\varphi}, \quad \mu \neq 0,
\end{gathered}
$$

the vacuum $\mathrm{BD}$ action goes into the action in the $\mathrm{EF}$ in the variables $\left(\tilde{g}_{\mu \nu}, \phi\right)$ as follows:

$$
S_{\mathrm{EF}}=\int d^{4} x \sqrt{-\widetilde{g}}\left[\widetilde{\mathbf{R}}-\mu \widetilde{g}_{\mu \nu} \phi_{, \mu} \phi_{, \nu}\right],
$$

where we have introduced a constant arbitrary parameter $\mu(= \pm 1)$ that can have any sign. The field equations from action (17), which are just Einstein's equations with source, are given by

$$
\begin{gathered}
\widetilde{R}_{\mu \nu}=\mu \phi_{, \mu} \phi_{, \nu}, \\
\square^{2} \phi=0 .
\end{gathered}
$$

We see that if the kinetic term $\mu g^{\mu v} \phi_{, \mu} \phi_{, \nu}$ in the action has an overall positive sign $(\mu=+1)$, the stresses satisfy all energy 
conditions. The counterpart of the singular Brans Class I solution (2) and (3) in the conformally rescaled EF thus is

$$
\begin{aligned}
d \tau_{\text {Buch }}^{2}= & -\left(\frac{1-m / 2 r}{1+B / 2 r}\right)^{2 \gamma} d t^{2} \\
& +\left(1-\frac{m}{2 r}\right)^{2(1+\gamma)}\left(1+\frac{m}{2 r}\right)^{2(1-\gamma)} \\
& \times\left[d r^{2}+r^{2} d \theta^{2}+r^{2} \sin ^{2} \theta d \psi^{2}\right], \\
\phi_{\text {Buch }}(r) & =\sqrt{\frac{2\left(1-\gamma^{2}\right)}{\mu}} \ln \left[\frac{1-m / 2 r}{1+m / 2 r}\right],
\end{aligned}
$$

where $\mu, m$, and $\gamma$ are arbitrary positive constants. This is known as Buchdahl solution [12]. The metric shares all the characters of the Class I solution. Though singular at $r=$ $m / 2$, the Buchdahl solution also yields the weak field solar system Robertson parameter values up to "one and half order" (see below) with the definition of $M=m \gamma$. At $\gamma=1$, Schwarzschild black hole is recovered. The result of Section 2 and the present one indicate that the local tests and the possibility of ultimate Schwarzschild limit go hand in hand with allowing a singularity in respective spacetimes.

If however the kinetic term $\mu g^{\mu \nu} \phi_{, \mu} \phi_{, \nu}$ has an overall negative sign $(\mu=-1)$, one has massless ghost matter source for which all the energy conditions including the dominant energy condition are violated giving rise to the possibility of wormholes. This is discovered by Ellis [13] and at the same time independently by Bronnikov [3]. For details about the relation between ghost and wormholes, see $[4,5]$. However, such wormholes are unstable with respect to linear perturbations $[14,15]$.

\section{Regular Wormhole in EF}

Choosing $\mu=-1$ and using (16), the metric in the EF becomes

$$
\begin{aligned}
d \tau_{\mathrm{EF}}^{2}= & \widetilde{g}_{\mu \nu} d x^{\mu} d x^{\nu} \\
= & -P\left(r^{\prime}\right) d t^{2}+Q\left(r^{\prime}\right) \\
& \times\left[d r^{\prime 2}+r^{\prime 2}\left(d \theta^{2}+\sin ^{2} \theta d \psi^{2}\right)\right],
\end{aligned}
$$

where

$$
\begin{gathered}
P\left(r^{\prime}\right)=\exp \left[2 \epsilon+4 \gamma \tan ^{-1}\left(\frac{r^{\prime}}{B^{\prime}}\right)\right], \\
Q\left(r^{\prime}\right)=\left(1+\frac{B^{\prime 2}}{r^{\prime 2}}\right)^{2} \exp \left[2 \zeta-4 \gamma \tan ^{-1}\left(\frac{r^{\prime}}{B^{\prime}}\right)\right], \\
\phi\left(r^{\prime}\right)=4 \lambda \tan ^{-1}\left(\frac{r^{\prime}}{B^{\prime}}\right), \quad \text { where } 2 \lambda^{2}=1+\gamma^{2} .
\end{gathered}
$$

Asymptotic flatness requires that $\epsilon=-\pi \gamma$ and $\zeta=\pi \gamma$. The constraint equation $2 \lambda^{2}=1+\gamma^{2}$ among free constants comes from the EF field equations when the solution is put into them. Note that unlike $\omega$ in Section 3, $\gamma$ here is unconstrained. The above solution is just a variant of the regular EllisBronnikov wormhole. ${ }^{4}$

Using the identity $\tan ^{-1}\left(r^{\prime} / B^{\prime}\right)=\pi / 2-\tan ^{-1}\left(B^{\prime} / r^{\prime}\right)$ for $r^{\prime}>0$, which corresponds to the positive mass mouth $M$ (= $2 B^{\prime} \gamma$ ), the metric functions (22) and (23) expand as

$$
\begin{aligned}
& P\left(r^{\prime}\right)=1-\frac{2 \alpha_{1} M}{r^{\prime}}+\frac{2 \beta_{1} M^{2}}{r^{\prime 2}}-\frac{3}{2} \epsilon_{1} \frac{M^{3}}{r^{\prime 3}}+\cdots, \\
& Q\left(r^{\prime}\right)=1+\frac{2 \gamma_{1} M}{r^{\prime}}+\frac{M^{2}}{r^{\prime 2}}\left(2+\frac{1}{2 \gamma^{2}}\right)+\cdots,
\end{aligned}
$$

giving values $\alpha_{1}=\beta_{1}=1, \gamma_{1}=1$, indicating that the solution yields exactly the same parametric values as in the vacuum Einstein theory but only up to the "one and half order." By that term, we mean first order in $M$ in $Q\left(r^{\prime}\right)$ and second order in $M$ in $P\left(r^{\prime}\right)$. The reason is that the parameter values $\alpha_{1}=\gamma_{1}=$ 1 are enough to explain the weak field light deflection, but the perihelion advance of planets in the solar system, though still a weak field test, requires information up to $M^{2}$ order in $P\left(r^{\prime}\right)$; that is, $\beta_{1}=1$. In that sense, this particular test might be termed as a "one and half order" test. One might wonder why the previous JF solutions (7)-(11) involving narrow $\omega$ do not support solar system tests at any order, while the present EF solutions (21)-(24) at least support them up to 1.5 order. In our opinion, this fact clearly underscores the fundamental distinction between Machian BD and Einstein theory at the level of regular solutions. No such distinction exists between singular solutions in either frame-both $\mathrm{BD}$ Class I and Buchdahl solutions support those tests to any order for arbitrarily large $\omega$ in the former and at $\gamma \rightarrow 1$ in the latter. These successes of the singular solutions led many to believe that it is only a matter of large $\omega$, the larger the value the closer it is to Einstein's theory; the impossibility of having large $\omega$ in the regular solution (Section 3 ) has gone unnoticed.

At the true second order, comparing the $M^{2}$ term in $Q\left(r^{\prime}\right)$ with the Robertson expansion (1), we see that

$$
\frac{3 \delta_{1} M^{2}}{2 r^{\prime 2}}=\frac{M^{2}}{r^{\prime 2}}\left(2+\frac{1}{2 \gamma^{2}}\right) \Longrightarrow \delta_{1}=\frac{4}{3}+\frac{1}{3 \gamma^{2}}
$$

In contrast, it can be verified that the Robertson expansion of singular Buchdahl $(M=m \gamma)$ solution (19) yields $\alpha_{1}=\beta_{1}=1$, $\gamma_{1}=1$, but at the second order gives

$$
\delta_{1}=\frac{4}{3}-\frac{1}{3 \gamma^{2}}
$$

which means that one recovers the other Schwarzschild value $\delta_{1}=1$ at $\gamma=1$. This Schwarzschild value is simply impossible to obtain in the regular Ellis-Bronnikov wormhole (21)-(24) in the EF that instead gives $\delta_{1}=5 / 3$ at $\gamma=1$. Hence, significant differences in prediction would appear in the second-order contribution distinguishing between singular and regular solutions in the EF. 


\section{Second Order Deflection}

We will start our discussion from the model independent Robertson expansion (1) with $A$ and $B$, generic of any spherically symmetric configuration. Now, for particles orbiting with velocity $V$ on the equatorial plane $(\theta=\pi / 2)$, the deflection angle measured by an asymptotic observer is given by $[10]$

$$
\alpha\left(r_{0}\right)=I\left(r_{0}\right)-\pi
$$

where $r_{0}$ is the distance of closest approach, and

$$
I\left(r_{0}\right)=2 \int_{r_{0}}^{\infty} \frac{d r}{r^{2}} A^{-(1 / 2)}(r)\left[\frac{1}{J^{2}}\left\{\frac{1}{B(r)}-E\right\}-\frac{1}{A r^{2}}\right]^{-(1 / 2)},
$$

where

$$
J=r_{0}\left[A\left(r_{0}\right)\left\{\frac{1}{B\left(r_{0}\right)}-E\right\}\right]^{1 / 2}, \quad E=1-V^{2} .
$$

Using the expressions for $A(r)$ and $B(r)$ from (1), the deflection for unbound particles up to the second-pN order, when $2 M \ll V^{2}$, becomes [16]

$$
\alpha\left(r_{0}\right)=a\left(\frac{M}{r_{0}}\right)+b\left(\frac{M}{r_{0}}\right)^{2}
$$

where

$$
\begin{gathered}
a=2\left(\gamma_{1}+\frac{1}{V^{2}}\right), \\
b=\frac{3 \pi \delta_{1}}{4}+\left(2+2 \gamma_{1}-\beta_{1}\right) \frac{\pi}{V^{2}}-2\left(\gamma_{1}+\frac{1}{V^{2}}\right)^{2} .
\end{gathered}
$$

For bound orbits, one would need to take into account also the effect of $\epsilon_{1}$, but we are focusing here only on the unbound orbits. For photon, $V=1$, and one obtains the known Schwarzschild expression at the values $\delta_{1}=\gamma_{1}=\beta_{1}=1$. However, there is great ambiguity in the literature about how the deflection variables are to be identified. For example, consider a light ray just grazing the limb of the sun. If the isotropic $r_{0}$ is identified with the independently measured Euclidean solar radius, the second-order contribution gives $\sim 3.5 \mu \mathrm{sec}$. If similar calculations are done in the standard Schwarzschild radial variable, say $\rho$, and identifying the distance of closest approach $\rho_{0}$ with the Euclidean solar radius, the second-order deflection will become $\sim 7 \mu \mathrm{sec}$. And finally, if the impact parameter $b$ is identified with the Euclidean solar radius, the contribution becomes $\sim 11 \mu \mathrm{sec}$ [16]. The reason for such ambiguity lies in the fact that Euclidean solar radius works well only up to first order, but not up to the second order as the error involved is also of the same order. To resolve the ambiguity, one has to regard $r_{0}$ or $\rho_{0}$ as another variable together with others like $\gamma_{1}$, $\beta_{1}$, and $\delta_{1}$ and compute them from the curve fitting to observed photon deflection data. Unfortunately, even this scheme would fail due to the fact that the light ray passing through $r=r_{0}$ (or $\rho=\rho_{0}$ ) would always follow the same trajectory giving the same deflection angle; there is no extra control parameter (since $V=1$ ) to regulate that angle. Thus massless unbound trajectories are of no use to measure the second-order deflection.

On the other hand, the deflection of a massive unbound particle trajectory can always be controlled by choosing different velocities $V<1$, and from curve fitting all the parameters $r_{0}, \gamma_{1}, \beta_{1}$, and $\delta_{1}$ can be unambiguously determined. The resulting parameter values can then be compared with the theoretical predictions of deflection from any spherically symmetric configuration, including those of Einstein or Brans-Dicke theory. It is exactly here that the upper limit on $\delta_{1}$ obtained from fitting can distinguish between the values in (26) and (27). Choose $\gamma=1$ in the solution. If it turns out from the fit that $\delta_{1} \simeq 1$, then we are possibly looking at a Schwarzschild black hole, and if $\delta_{1} \simeq$ $5 / 3$, then we are looking at the Ellis-Bronnikov wormhole. A very speculative natural scenario for the observation of material orbit deflection could be provided by the trajectory of unstable $\mathrm{H} 1$ orbits with nonzero angular momentum moving in and out to asymptotes enclosing the galactic center. Of course, it is technically a very challenging task. ${ }^{5}$

\section{Conclusion}

The contents of Section 2 show that, in singular BD I, $\omega$ can assume any value leading to the Schwarzschild limit at $\omega \rightarrow$ $\pm \infty$. On the other hand, we have just seen in Section 3 that the regular solution (7)-(11) allows only to narrow a range $-2<\omega<-3 / 2$, which prevents any passage to solar system values as is evident from the Robertson parameters. It is also evident that the solution has no horizon, so it cannot tend to a black hole. However, the spacetime can still represent a very different object, a traversable wormhole. (For a classic treatise on wormholes, see [17].) This wormhole structure occurs naturally, in both JF and EF, as opposed to many artificially engineered wormholes presented in the literature. (By natural, we mean a well-defined Lagrangian theory.) In the EF (Section 5), which is just Einstein's general relativity, the wormhole spacetime yields even the known weak field solar system parameters up to 1.5 order, but significant differences appear in the Robertson parameter $\delta_{1}$. While in the singular Buchdahl solution (Section 4), one can reduce $\delta_{1}$ to the Schwarzschild value of unity; there is no chance to do so in the case of wormhole solution. This could in principle mean a decisive test about the observable signature of the nonsingular Ellis-Bronnikov wormhole vis-a-vis the singular Buchdahl solution (Section 6). We are aware that instability of wormhole solutions in the massless minimally coupled theory $[14,15]$ spoils any chance of their very physical existence, let alone the prospect of such measurements. However, our aim was to point out the theoretical distinctions between different kinds of geometric structures as exposed by the Robertson expansion. Note also that we had not entered the issue as to which frame JF or EF is physical. For informative discussions on this issue, see, for example, [1822]. 
Finally, we return to the topic in the title. The answer seems to be largely a matter of choice. Depending on the attitude, we might accept the singular Class I solution as the solution prizing its weak field successes. Or we may accept the nonsingular Class II solution as the solution, which would then amount to simply denying vacuum Brans-Dicke theory of its observational successes because of narrow range of $\omega$. But it would nonetheless offer us a natural traversable wormhole. The third option is to accept both the solutions (BD I and II) as describing two different scenarios, but Schwarschild black holes are certainly ruled out in either frame since the curvatures diverge in BD I and no horizon in BD II. Since neither black holes nor wormholes have been conclusively observed, we think that the best option is to keep the choice open. Dismissing the vacuum BD theory as effectively nondistinct from Einstein theory because of large $\omega$ would blind us to the distinct geometric structures discussed here.

\section{Endnotes}

1. Ernst Mach, a nineteenth century philosopher, argued that local inertial properties of matter are determined by the matter distribution in the whole universe. This is known as Mach's principle.

2. In the $\mathrm{EF}$, vacuum $\mathrm{BD}$ equations reduce to minimally coupled scalar field Einstein theory. Since introducing scalar fields into many theories is the order of the day, it will be useful to see how the regular solutions behave in the simplest scalar field gravity theory.

3. The reverse route (Class II $\rightarrow$ Class I) was first shown by Bhadra and Sarkar [23] with the conclusion that the two solutions are not independent. However, the detailed wormhole features of their seed Class II were not investigated.

4. To put the solution into a more familiar form, transform the radial variable as

$$
\ell=r^{\prime}-\frac{B^{2}}{r^{\prime}}
$$

With $B^{\prime}=m / 2$, and using the identity

$$
2 \tan ^{-1}\left(\frac{\ell+\sqrt{\ell^{2}+m^{2}}}{m}\right) \equiv \frac{\pi}{2}+\tan ^{-1}\left(\frac{\ell}{m}\right)
$$

we see that the solution set (21)-(24) becomes just the Ellis-Bronnikov (EB) wormhole as follows:

$$
\begin{aligned}
& d \tau_{\mathrm{EB}}^{2}=-F_{\mathrm{EB}} d t^{2}+F_{\mathrm{EB}}^{-1} \\
& \times\left[d \ell^{2}+\left(\ell^{2}+m^{2}\right)\left(d \theta^{2}+\sin ^{2} \theta d \psi^{2}\right)\right] \\
& F_{\mathrm{EB}}=\exp \left[-\pi \gamma+2 \gamma \tan ^{-1}\left(\frac{\ell}{m}\right)\right] \\
& \phi_{\mathrm{EB}}=\lambda\left[\pi+2 \tan ^{-1}\left(\frac{\ell}{m}\right)\right], \quad 2 \lambda^{2}=1+\gamma^{2} .
\end{aligned}
$$

5. We are thankful to Bhadra et al. for pointing out that the deflection of ionized hydrogen by the intergalactic magnetic field would mask the small second-order contribution. However, the realization that the deflection data, not of light but of only unbound material orbits, can allow us to test the second-order contribution is very fundamental and was first advanced in [16]. All planned experiments to measure that contribution should take into account this proposal.

\section{Acknowledgment}

The authors thank Sonali Sarkar for technical assistance with some of the past literature.

\section{References}

[1] K. Gödel, "An example of a new type of cosmological solutions of einstein's field equations of gravitation," Reviews of Modern Physics, vol. 21, pp. 447-450, 1949.

[2] C. H. Brans and R. H. Dicke, "Mach's principle and a relativistic theory of gravitation," Physical Review, vol. 124, no. 3, pp. 925935, 1961.

[3] K. A. Bronnikov, Acta Physica Polonica B, vol. 4, p. 251, 1973.

[4] K. A. Bronnikov, M. V. Skvortsova, and A. A. Starobinsky, "Notes on wormhole existence in scalar-tensor and $\mathrm{F}(\mathrm{R})$ gravity," Gravitation and Cosmology, vol. 16, no. 3, pp. 216-222, 2010.

[5] K. A. Bronnikov and A. A. Starobinsky, "No realistic wormholes from ghost-free scalar-tensor phantom dark energy," JETP Letters, vol. 85, no. 1, pp. 1-5, 2007.

[6] C. H. Brans, "Mach's principle and a relativistic theory of gravitation. II," Physical Review, vol. 125, no. 6, pp. 2194-2201, 1962.

[7] A. Bhadra and K. K. Nandi, " $\omega$ dependence of the scalar field in Brans-Dicke theory," Physical Review D, vol. 64, Article ID 087501, 3 pages, 2001.

[8] K. K. Nandi, I. Nigmatzyanov, R. Izmailov, and N. G. Migranov, "New features of extended wormhole solutions in the scalar field gravity theories," Classical and Quantum Gravity, vol. 25, no. 16, Article ID 165020, 2008.

[9] A. Bhattacharya, R. Izmailov, E. Laserra, and K. K. Nandi, "A nonsingular Brans wormhole: an analogue to naked black holes," Classical and Quantum Gravity, vol. 28, Article ID 155009, 2011.

[10] S. Weinberg, Gravitation and Cosmology, John Wiley \& Sons, New York, NY, USA, 1972.

[11] C. W. Misner, K. S. Thorne, and J. A. Wheeler, Gravitation, W. H. Freeman, San Francisco, Calif, USA, 1973.

[12] H. A. Buchdahl, "Reciprocal static metrics and scalar fields in the general theory of relativity," Physical Review, vol. 115, no. 5, pp. 1325-1328, 1959.

[13] H. G. Ellis, "Ether flow through a drainhole: a particle model in general relativity," Journal of Mathematical Physics, vol. 14, p. 104, 1973, Erratum in Journal of Mathematical Physics, vol. 15, p. $520,1974$.

[14] J. A. Gonzalez, F. S. Guzmán, and O. Sarbach, "Instability of wormholes supported by a ghost scalar field: I. Linear stability analysis," Classical and Quantum Gravity, vol. 26, Article ID 015010, 2009. 
[15] J. A. Gonzalez, F. S. Guzmán, and O. Sarbach, "Instability ofwormholes supported by a ghost scalar field: II. Nonlinear evolution," Classical and Quantum Gravity, vol. 26, Article ID 015011, 2009.

[16] A. Bhadra, K. Sarkar, and K. K. Nandi, "Testing gravity at the second post-Newtonian level through gravitational deflection of massive particles," Physical Review D, vol. 75, Article ID 123004, 2007.

[17] M. Visser, Lorentzian Wormholes-From Einstein to Hawking, AIP, New York, NY, USA, 1995.

[18] É.É. Flanagan, “The conformal frame freedom in theories of gravitation," Classical and Quantum Gravity, vol. 21, pp. 38173829, 2004.

[19] J. E. Lidsey, D. Wands, and E. J. Copeland, "Superstring cosmology," Physics Report, vol. 337, no. 4-5, pp. 343-492, 2000.

[20] M. Gasperini and G. Veneziano, "The pre-big bang scenario in string cosmology," Physics Report, vol. 373, no. 1-2, pp. 1-212, 2003.

[21] V. Faraoni, "The $\omega \rightarrow \infty$ limit of Brans-Dicke theory," Physics Letters A, vol. 245, pp. 26-30, 1998.

[22] A. Bhadra, K. Sarkar, D. P. Datta, and K. K. Nandi, "Brans-Dicke theory: jordan versus einstein frame," Modern Physics Letters A, vol. 22, no. 5, pp. 367-375, 2007.

[23] A. Bhadra and K. Sarkar, "On static spherically symmetric solutions of the vacuum Brans-Dicke theory," General Relativity and Gravitation, vol. 37, pp. 2189-2199, 2005. 

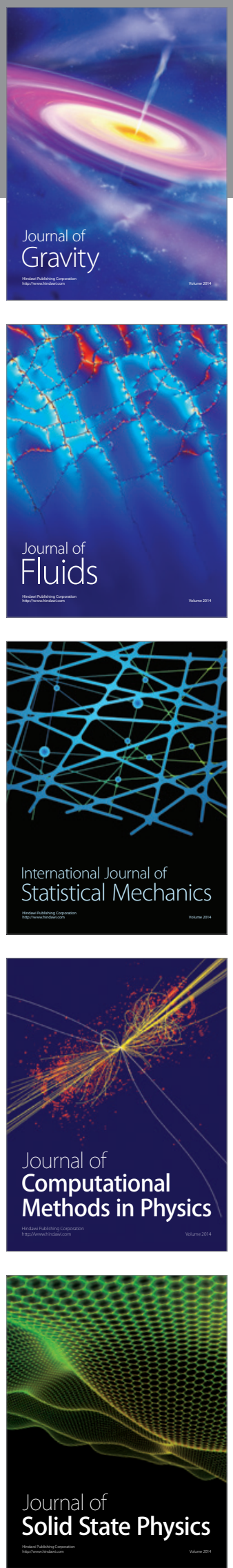

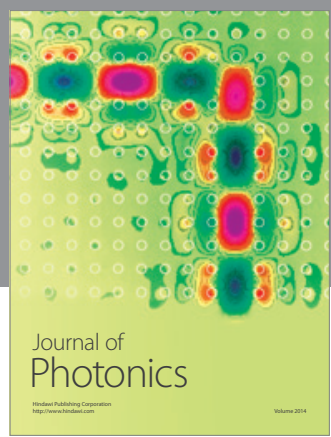

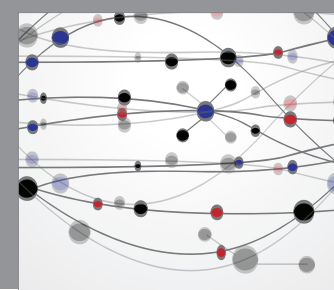

The Scientific World Journal

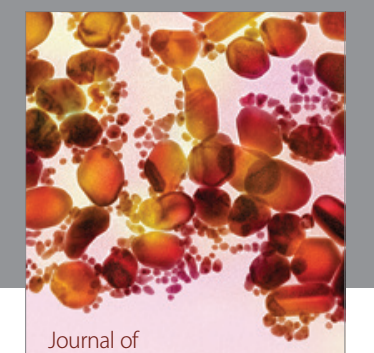

Soft Matter
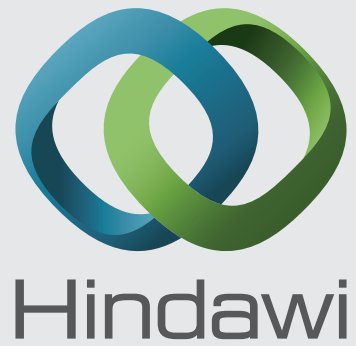

Submit your manuscripts at

http://www.hindawi.com
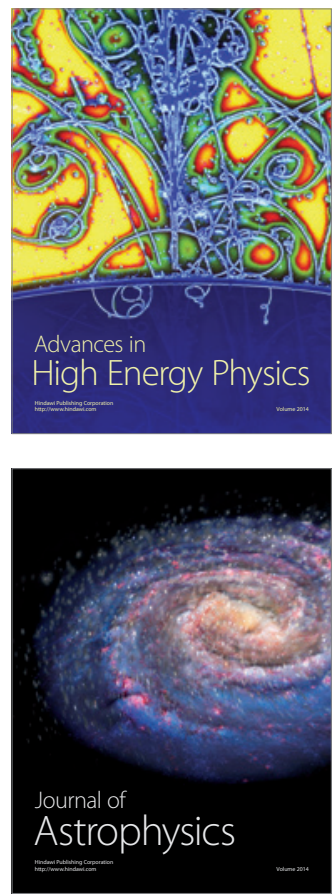
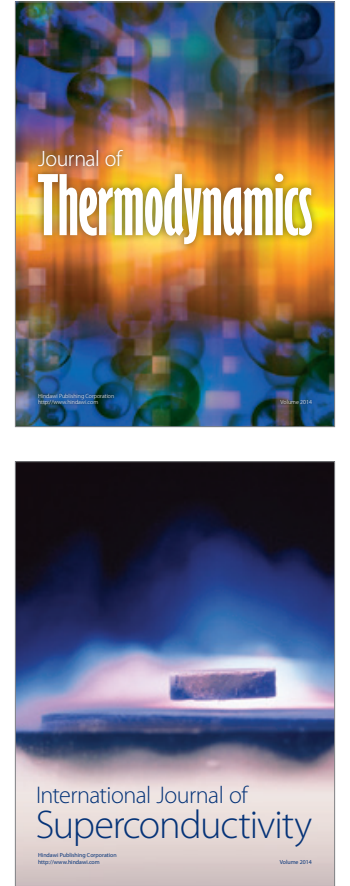
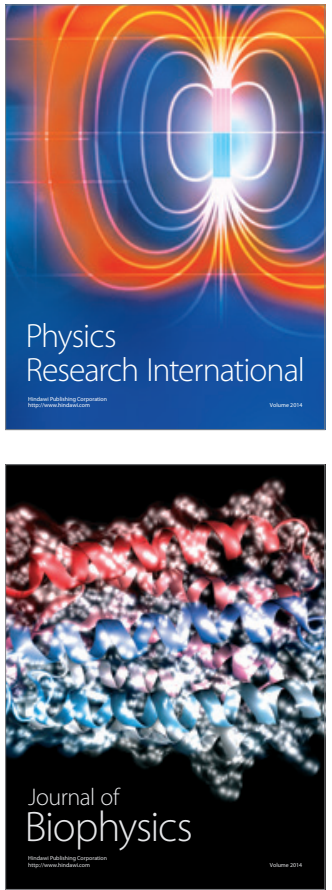
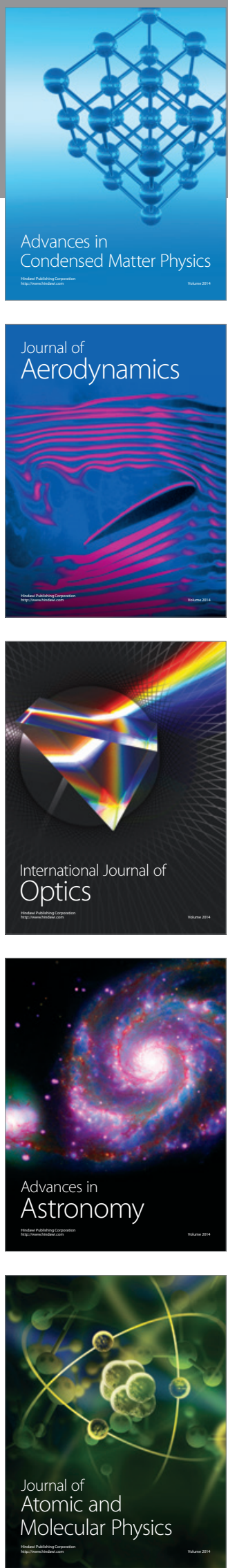